\title{
BALANCE OF GREEN OPEN SPACE TO SUPPORT GREEN SETTLEMENT IN YOGYAKARTA URBAN AREA
}

\author{
Widodo Brontowiyono \\ Department of Environmental Engineering \\ Faculty of Civil Engineering and Planning, Universitas Islam Indonesia (UII) \\ Jl. Kaliurang Km 14,5 Yogyakarta 55584 \\ E-mail: widodo.bronto@gmail.com
}

\begin{abstract}
The greatest problem relating to settlement growth in Yogyakarta urban area is the declining number of green open spaces. This study aimed to analyze the balance between the availability of and need for green open space development to promote the existence of green settlement in Yogyakarta urban area. The methods employed in this study were field research and studio analysis by referring to the related research methods previously conducted by the researcher as well as by the others. The results showed that the extent of green open space in Yogyakarta urban area reached 1,469.45 Ha or 16.2\% of the total area. Therefore, the need for green open space lacked $13.8 \%$ of the total area. The general condition of the green open space was categorized as average in either housing clusters or settlements (non-housing clusters). Good condition was found only in low-density settlement, while poor condition of green open space was located in high-density settlement. The development of green open space should focus on public green open space while maintaining the private one. Some of the potential areas for this included rice fields, village treasury lands, riverbanks, roadsides, railway boundaries, public areas, and others.
\end{abstract}

Keywords: development, green open space, green settlement, Yogyakarta

\section{INTRODUCTION}

The availability of green open space (GOS) is one of the important factors for urban microclimate. The average temperature of urban area is 40C higher than that of the suburban (Widodo B. et al, 2011). This indicates the decreasing quality of open space in urban area due to imbalanced condition between built-up area and non-built-up area.

According to the UN projection, three out of five world inhabitants will live in urban area by 2030 (Baiquni, 2002). Yogyakarta urban area (YUA) has experienced a significant level of urbanization. The available area is limited, forcing urbanization in Yogyakarta to spread across Sleman Regency and Bantul Regency. Consequently, settlement area becomes broader and denser. Widodo B. (2005) and Widodo B. et al (2009b) predicted that rice fields in Sleman, Yogyakarta, and Bantul would be completely used for settlement by 2030s if no control was taken.

The more complex urban dynamics have become a tough challenge to sustainable development. The urban physical area keeps being filled with buildings, surface of both building roofs and yards is 
hardened, and green open space continues to narrow or even almost to disappear. The urban environment tends to develop economically but to decline ecologically (Harjito, D.A., 2007). One of the impacts is an ambient temperature raise that will trigger urban heat island - though on a small scale (Li et al., 2005). On the other hand, global warming issue continues to frighten the world.

The most effective strategy to mitigate the threat of global warming is improving the condition of local environment (Widodo B. et al, 2009a). Widodo B et al (2011) and Dinas Kimpraswil DIY (2006) suggested two main principles to overcome urban heat island, which are to shade hardened surface in public spaces and to sustain the flow of winds. Law No. 26 Year 2007 on Spatial Planning has also mandated urban area to provide GOS with a minimum of $30 \%$ of the total extent, consisting of $20 \%$ public GOS and $10 \%$ private GOS. Micro climate change is an environmental hazard and has the potential to bring disasters (Coburn, 1994; Reed, 1995). One of the efforts to prevent disasters can include law enforcement. In the context of micro climate change relating to green open space, the prevention can optimize the mandate of Law No. 26 Year 2007 on Spatial Planning (Undang-Undang Republik Indonesia Nomor 26 Tahun 2007 Tentang Penataan Ruang) to provide at least $30 \%$ of urban area for GOS.

The major issue around settlement growth in Yogyakarta urban area is the decreasing green open space. Therefore, this study attempted to map the potential and to design strategies for the development of green open space to encourage the establishment of green settlement in Yogyakarta urban area.

This research aimed to: identify the existing green open space in Yogyakarta urban area, analyze the need for green open space in Yogyakarta urban area and analyze the development of green open space in settlement area.

\section{RESEARCH METHODS}

\subsection{Data Collection Techniques}

The socio-economic data were collected primarily and secondarily. The secondary data were gathered from the reports or other official sources of related institutions. Meanwhile, the primary data were collected through semi-structured interview with questionnaire. In addition, the physical data of GOS were obtained from field survey, semi-structured interview with questionnaire, as well as in-depth interview. The survey results were in the form of documentation photos, sketches, numerical figures, and others.

The research population involved households living in Yogyakarta urban area. The respondents were the patriarchs selected through proportional cluster random sampling. The settlement clusters 
were divided into the settlement area in large housing complex (elite), medium housing complex, small housing complex, and urban kampong. The total samples used in this study were 100 patriarchs.

\subsection{Data Processing and Analysis}

\subsubsection{Analysis of the existing GOS condition}

The analysis of existing condition of GOS in each settlement cluster was conducted for the following aspects:

1. Tree density or canopy volume

The analysis was performed by interpreting the satellite imagery from GIS-based Landsat 2016.

2. Evaluation of GOS quality

An evaluative analysis was conducted through a field survey of GOS availability. The observation result was then compared to the standard of infrastructure for environmental conservation. The analysis results consisted of three (3) criteria, including environmentally friendly (good), less environmentally friendly (average), and not environmentally friendly (poor). The criteria and data gathering techniques are presented in the following table.

Table 1. Criteria and Data Collection Techniques for Settlement Evaluation

\begin{tabular}{|c|c|c|c|}
\hline \multicolumn{3}{|c|}{ Condition } & \multirow{2}{*}{$\begin{array}{l}\text { Collection } \\
\text { Technique }\end{array}$} \\
\hline Good & Average & Poor & \\
\hline $\begin{array}{l}\text { Owning } 20 \% \text { total } \\
\text { area for private } \\
\text { GOS }\end{array}$ & $\begin{array}{l}\text { Owning } 10-20 \% \\
\text { total area for } \\
\text { private GOS }\end{array}$ & $\begin{array}{l}\text { Owning less than } \\
10 \% \text { total area for } \\
\text { private GOS }\end{array}$ & questionnaire \\
\hline $\begin{array}{l}\text { Owning } 10 \% \text { total } \\
\text { area for public } \\
\text { GOS }\end{array}$ & $\begin{array}{l}\text { Owning } 5-10 \% \\
\text { total area for public } \\
\text { GOS }\end{array}$ & $\begin{array}{l}\text { Owning less than } \\
5 \% \text { total area for } \\
\text { public GOS }\end{array}$ & $\begin{array}{l}\text { observation/ } \\
\text { questionnaire }\end{array}$ \\
\hline
\end{tabular}

Source: Kementerian Negara Perumahan Rakyat, 2008 (with modification)

\subsubsection{Analysis of the need for GOS}

Analysis of the need for or shortage of GOS was performed based on the requirement of area in accordance with the regulation. Law No. 26 Year 2007 on Spatial Planning regulated that the proportion of green open space in urban area is at least $30 \%$ (thirty percent) of the total city area with $20 \%$ area for public and $10 \%$ extent for private.

The need for GOS = the total need for GOS - the availability of GOS 


\section{RESULTS AND DISCUSSION}

\subsection{Existing Condition of GOS}

The identification of GOS existing condition used GIS technique and field survey. Figure 4.1 illustrates the distribution of existing GOS in Yogyakarta urban area according to the Landsat8 Satellite Imagery year 2016. The detail of distribution area is presented in Table 2. In general, the GOS in Yogyakarta urban area reached 1,469.45 Ha or $16.2 \%$ of the total area.

Table 2. Extent of Green Open Space in Yogyakarta Urban Area

\begin{tabular}{|c|c|c|c|c|c|}
\hline \multirow{2}{*}{$\begin{array}{l}\text { Regency/ } \\
\text { City }\end{array}$} & \multirow{2}{*}{ District } & \multirow{2}{*}{$\begin{array}{l}\text { Village/Urban } \\
\text { Community }\end{array}$} & \multirow{2}{*}{ Area (Ha) } & \multicolumn{2}{|c|}{ Existing GOS } \\
\hline & & & & $\mathbf{H a}$ & $\%$ \\
\hline \multirow[t]{11}{*}{ Sleman } & Depok & Caturtunggal & 523.14 & 157.17 & $30.04 \%$ \\
\hline & & Condongcatur & 448.57 & 113.41 & $25.28 \%$ \\
\hline & & Maguwoharjo & 694.82 & 126.31 & $18.18 \%$ \\
\hline & Gamping & Ambarketawang & 336.00 & 38.48 & $11.45 \%$ \\
\hline & & Banyuraden & 232.20 & 29.94 & $12.89 \%$ \\
\hline & & Nogotirto & 329.12 & 33.40 & $10.15 \%$ \\
\hline & & Trihanggo & 554.34 & 39.62 & $7.15 \%$ \\
\hline & Mlati & Sendangadi & 306.10 & 42.54 & $13.90 \%$ \\
\hline & & Sinduadi & 385.42 & 78.35 & $20.33 \%$ \\
\hline & Ngaglik & Sariharjo & 398.69 & 79.77 & $20.01 \%$ \\
\hline & & Total & $4,208.40$ & 738.99 & $17.56 \%$ \\
\hline \multirow[t]{21}{*}{ Yogyakarta } & Danurejan & Bausasran & 35.18 & 3.65 & $10.37 \%$ \\
\hline & & Suryatmajan & 24.15 & 4.47 & $18.53 \%$ \\
\hline & & Tegalpanggung & 30.00 & 3.50 & $11.66 \%$ \\
\hline & Gondokusuman & Terban & 55.72 & 6.02 & $10.81 \%$ \\
\hline & & Baciro & 78.47 & 9.78 & $12.46 \%$ \\
\hline & & Demangan & 51.67 & 9.44 & $18.27 \%$ \\
\hline & & Klitren & 57.57 & 7.09 & $12.32 \%$ \\
\hline & & Kotabaru & 52.92 & 11.27 & $21.29 \%$ \\
\hline & Gondomanan & Prawirodirjan & 24.06 & 4.64 & $19.28 \%$ \\
\hline & & Ngupasan & 55.20 & 10.93 & $19.79 \%$ \\
\hline & & Prawirodirjan & 24.06 & 4.64 & $19.28 \%$ \\
\hline & Gedongtengen & Pringgokusuman & 43.12 & 3.81 & $8.83 \%$ \\
\hline & & Sosromenduran & 39.81 & 5.59 & $14.04 \%$ \\
\hline & Jetis & Bumijo & 45.69 & 6.44 & $14.09 \%$ \\
\hline & & Cokrodiningratan & 37.51 & 5.83 & $15.55 \%$ \\
\hline & & Gowongan & 37.37 & 6.26 & $16.74 \%$ \\
\hline & Kotagede & Prenggan & 44.16 & 8.12 & $18.40 \%$ \\
\hline & & Rejowinangun & 54.71 & 12.20 & $22.30 \%$ \\
\hline & & Purbayan & 21.10 & 3.17 & $15.02 \%$ \\
\hline & Kraton & Kadipaten & 22.39 & 5.11 & $22.84 \%$ \\
\hline & & Panembahan & 35.23 & 9.54 & $27.09 \%$ \\
\hline
\end{tabular}




\begin{tabular}{|c|c|c|c|c|c|}
\hline \multirow{3}{*}{$\begin{array}{l}\text { Regency/ } \\
\text { City }\end{array}$} & \multirow{2}{*}{ District } & \multirow{2}{*}{$\begin{array}{l}\text { Village/Urban } \\
\text { Community }\end{array}$} & \multirow{2}{*}{ Area (Ha) } & \multicolumn{2}{|c|}{ Existing GOS } \\
\hline & & & & Ha & $\%$ \\
\hline & & Patehan & 21.59 & 5.69 & $26.37 \%$ \\
\hline & \multirow[t]{3}{*}{ Mantrijeron } & Gedongkiwo & 37.58 & 6.15 & $16.37 \%$ \\
\hline & & Mantrijeron & 42.09 & 8.40 & $19.96 \%$ \\
\hline & & Suryodiningratan & 34.37 & 5.33 & $15.50 \%$ \\
\hline & \multirow[t]{3}{*}{ Mergangsan } & Brontokusuman & 38.58 & 10.48 & $27.15 \%$ \\
\hline & & Keparakan & 22.61 & 3.37 & $14.89 \%$ \\
\hline & & Wirogunan & 40.51 & 7.81 & $19.27 \%$ \\
\hline & \multirow[t]{2}{*}{ Ngampilan } & Ngampilan & 34.42 & 2.83 & $8.22 \%$ \\
\hline & & Notoprajan & 21.63 & 4.05 & $18.72 \%$ \\
\hline & \multirow[t]{2}{*}{ Pakualaman } & Gunungketur & 23.07 & 3.42 & $14.82 \%$ \\
\hline & & Purwokinanti & 29.31 & 2.85 & $9.71 \%$ \\
\hline & \multirow[t]{4}{*}{ Tegalrejo } & Karangwaru & 35.06 & 3.96 & $11.29 \%$ \\
\hline & & Bener & 22.93 & 3.50 & $15.26 \%$ \\
\hline & & Kricak & 30.36 & 3.09 & $10.18 \%$ \\
\hline & & Tegalrejo & 30.72 & 7.56 & $24.62 \%$ \\
\hline & \multirow[t]{7}{*}{ Umbulharjo } & Giwangan & 40.16 & 10.80 & $26.88 \%$ \\
\hline & & Mujamuju & 66.02 & 11.22 & $16.99 \%$ \\
\hline & & Pandeyan & 55.79 & 15.17 & $27.19 \%$ \\
\hline & & Semaki & 43.16 & 7.95 & $18.43 \%$ \\
\hline & & Tahunan & 35.60 & 7.27 & $20.41 \%$ \\
\hline & & Warungboto & 37.02 & 5.45 & $14.72 \%$ \\
\hline & & Sorosutan & 56.65 & 13.02 & $22.98 \%$ \\
\hline & \multirow[t]{4}{*}{ Wirobrajan } & Pakuncen & 30.63 & 6.87 & $22.43 \%$ \\
\hline & & Patangpuluhan & 25.03 & 4.43 & $17.70 \%$ \\
\hline & & Wirobrajan & 31.74 & 4.23 & $13.32 \%$ \\
\hline & & Total & $1,756.75$ & 306.37 & $17.44 \%$ \\
\hline \multirow[t]{11}{*}{ Bantul } & \multirow[t]{6}{*}{ Banguntapan } & Banguntapan & 328.44 & 44.56 & $13.57 \%$ \\
\hline & & Baturetno & 331.06 & 53.86 & $16.27 \%$ \\
\hline & & Jagalan & 11.19 & 1.42 & $12.69 \%$ \\
\hline & & Singosaren & 63.56 & 43.02 & $67.68 \%$ \\
\hline & & Tamanan & 312.26 & 22.33 & $7.15 \%$ \\
\hline & & Wirokerten & 282.22 & 19.06 & $6.75 \%$ \\
\hline & \multirow[t]{3}{*}{ Kasihan } & Ngestiharjo & 249.06 & 48.31 & $19.40 \%$ \\
\hline & & Tamantirto & 344.27 & 50.45 & $14.65 \%$ \\
\hline & & Tirtonirmolo & 258.75 & 49.67 & $19.20 \%$ \\
\hline & \multirow[t]{2}{*}{ Sewon } & Panggungharjo & 389.84 & 46.79 & $12.00 \%$ \\
\hline & & Bangunharjo & 578.65 & 44.62 & $7.71 \%$ \\
\hline \multirow{2}{*}{\multicolumn{3}{|c|}{ Yogyakarta Urban Area (KPY) }} & $3,149.30$ & 424.09 & $13.47 \%$ \\
\hline & & & $9,114.46$ & $1,469.45$ & $16.12 \%$ \\
\hline
\end{tabular}


Figure 1. Map of Existing Green Open Space in Yogyakarta Urban Area

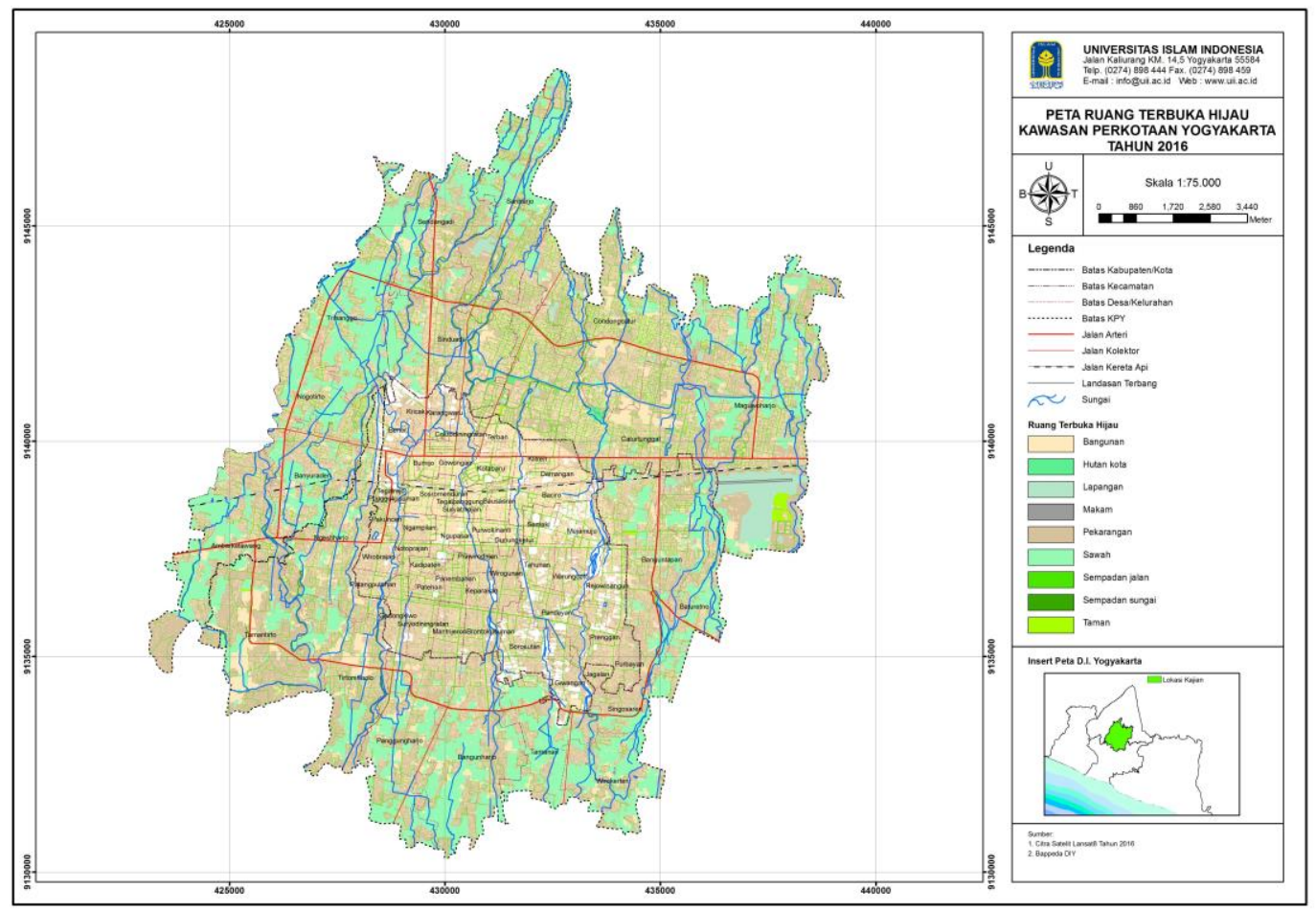

\subsubsection{GOS of Housing Clusters}

\section{Large Housing Complex (Elite)}

The result of field survey analysis for the quality of GOS in large housing complex (elite) is presented in Table 3. The table shows that, in general, the conservation effort through GOS has been made with average quality.

Table 3. Quality of GOS in Elite Housing Complex

\begin{tabular}{clc}
\hline No & Name of Housing Complex & Condition \\
\hline 1 & The Paradise & average \\
2 & North Hill Residence & average \\
3 & Mataram Bumi Sejahtera & average \\
4 & Pondok Kadipiro Permai & average \\
5 & Timoho Town House & average \\
6 & Kusuma Negara Town House & average \\
7 & Semaki Cluster & average \\
8 & Tiara Mas Wonocatur & average \\
9 & Grinhos & average \\
10 & De Asmarandana Resident & average \\
& Mean & average \\
\hline
\end{tabular}

Source: Field Survey (2016) 


\section{Medium Housing Complex}

The evaluation of GOS quality is illustrated in Table 4. This table indicates the condition of environmental conservation facility with average quality. Good condition was located in only one housing complex.

Table 4. Quality of GOS in Medium Housing Complex

\begin{tabular}{cll}
\hline No & \multicolumn{1}{c}{$\begin{array}{c}\text { Name of Housing } \\
\text { Complex }\end{array}$} & \multicolumn{1}{c}{ Quality of GOS } \\
\hline 1 & Pondok Permai Palagan & average \\
2 & Citra Nirwana & average \\
3 & Pesona Kuantan & average \\
4 & Harmoni Graha Gemilang & average \\
5 & Timoho Asri 3 & good \\
6 & Timoho Asri 1 & average \\
7 & Mutiara Town House & average \\
8 & Metro Harmony Residence & average \\
& Mean & average \\
\hline
\end{tabular}

Source: Field Survey (2016)

\section{Small Housing Complex}

The result of quality evaluation can be seen in the following Table 5 .

Table 5. Quality of GOS in Small Housing Complex

\begin{tabular}{cll}
\hline No & \multicolumn{1}{c}{$\begin{array}{c}\text { Name of Housing } \\
\text { Complex }\end{array}$} & $\begin{array}{c}\text { Quality of } \\
\text { GOS }\end{array}$ \\
\hline 1 & Griya Nganti Asri & average \\
2 & Graha Adi Pratama & good \\
3 & Griya surya asri & good \\
4 & Taman Mas 2 & average \\
5 & Tamantirto Asri 1 & good \\
6 & PPLH Gunung Sempu & average \\
7 & Bumi Tirto Indah & good \\
8 & Griya Tirtonirmolo Asri & good \\
9 & Nyoto Asri 3 & average \\
10 & Purimas Citra Gemilang & average \\
11 & Metro Harmony & average \\
& Residence & \\
& Mean & average \\
\hline
\end{tabular}

Source: Field Survey (2016) 
Table 5 indicates that in general the GOS was of average quality. Good condition existed in six housing complexes $(45.55 \%)$.

\subsubsection{GOS of Non-Housing Complex Clusters}

\section{High-Density Settlement}

The result of GOS quality evaluation is described in Table 6. It shows that the GOS availability was in poor condition.

Table 6. Quality of GOS in High-Density Settlement

\begin{tabular}{cll}
\hline No & \multicolumn{1}{c}{$\begin{array}{c}\text { Village/Urban } \\
\text { Community }\end{array}$} & Quality of GOS \\
\hline 1 & Notoprajan & poor \\
2 & Panggungharjo & poor \\
3 & Ngestiharjo & poor \\
4 & Caturtunggal & poor \\
& Mean & poor \\
\hline
\end{tabular}

Source: Field Survey (2016)

\section{Medium-Density Settlement}

The evaluation result of GOS quality is illustrated in Table 7 indicating that the GOS was of average quality.

Table 7. Quality of GOS in Medium-Density Settlement

\begin{tabular}{cll}
\hline No & \multicolumn{1}{c}{$\begin{array}{c}\text { Village/Urban } \\
\text { Community }\end{array}$} & Quality of GOS \\
\hline 1 & Baciro & average \\
2 & Kricak & average \\
3 & Karangwaru & average \\
4 & Prawirodirjan & average \\
5 & Baturetno & good \\
6 & Wirokerten & average \\
7 & Tamanan & average \\
8 & Sinduharjo & good \\
9 & Minomartani & average \\
10 & Sariharjo & average \\
& Mean & average \\
\hline
\end{tabular}

Source: Field Survey (2016)

\section{Low-Density Settlement}

The respondents' information on the GOS condition is described in Table 8. This table indicates that the quality of GOS was average. 
Table 8. Quality of GOS in Low-Density Settlement

\begin{tabular}{cll}
\hline No & \multicolumn{1}{c}{$\begin{array}{c}\text { Village/Urban } \\
\text { Community }\end{array}$} & Quality of GOS \\
\hline 1 & Patehan & good \\
2 & Semaki & good \\
3 & Kotabaru & good \\
4 & Tegal Panggung & good \\
5 & Gunungketur & good \\
6 & Bener & good \\
7 & Jagalan & good \\
8 & Condongcatur & good \\
9 & Sendangadi & good \\
10 & Sinduadi & good \\
& Mean & good \\
\hline
\end{tabular}

Source: Field Survey (2016)

All the above results of evaluative analysis are summarized in Table 9. On a macro scale, both the housing clusters and non-housing settlements have the average GOS quality. This evaluation becomes the basis for the selection of GOS management and supply model.

Table 9. Recapitulation of GOS Quality in Settlements

\begin{tabular}{ll}
\hline \multicolumn{1}{c}{ Type of Settlement } & \multicolumn{1}{c}{$\begin{array}{c}\text { Quality of } \\
\text { GOS }\end{array}$} \\
\hline Elite Housing & average \\
Medium Housing & average \\
Small Housing & average \\
Mean for Housing & average \\
High-Density Settlement & Poor \\
Medium-Density & average \\
Settlement & \\
Low-Density Settlement & good \\
Mean for Settlement & average \\
Total Mean & average \\
\hline
\end{tabular}

\subsubsection{Need for GOS}

Table 10. Extent of Need for and Balance of Green Open Space in Yogyakarta Urban Area

\begin{tabular}{lllrrr}
\hline \multirow{2}{*}{ Regency/City } & \multirow{2}{*}{ District } & Village/Urban & \multirow{2}{*}{$\begin{array}{c}\text { Ideal need } \\
\text { for GOS }\end{array}$} & & \multicolumn{2}{c}{ Surplus/Deficit RTH } \\
\cline { 6 - 6 } & & Community & (Ha) & \multicolumn{1}{c}{ Ha } & \multicolumn{1}{c}{$\%$} \\
\hline Sleman & Depok & Caturtunggal & 156.94 & 0.23 & $0.04 \%$ \\
& & Condongcatur & 134.57 & -21.16 & $-4.72 \%$ \\
& \multirow{3}{*}{ Gamping } & Maguwoharjo & 208.45 & -82.14 & $-11.82 \%$ \\
& & Ambarketawang & 100.80 & -62.32 & $-18.55 \%$ \\
& & Banyuraden & 69.66 & -39.72 & $-17.11 \%$ \\
& Nogotirto & 98.74 & -65.34 & $-19.85 \%$ \\
& & Trihanggo & 166.30 & -126.68 & $-22.85 \%$ \\
\hline
\end{tabular}




\begin{tabular}{|c|c|c|c|c|c|}
\hline \multirow{2}{*}{ Regency/City } & \multirow{2}{*}{ District } & \multirow{2}{*}{$\begin{array}{c}\text { Village/Urban } \\
\text { Community }\end{array}$} & \multirow{2}{*}{$\begin{array}{c}\text { Ideal need } \\
\text { for GOS } \\
\text { (Ha) }\end{array}$} & \multicolumn{2}{|c|}{ Surplus/Deficit RTH } \\
\hline & & & & $\mathbf{H a}$ & $\%$ \\
\hline & Mlati & Sendangadi & 91.83 & -49.29 & $-16.10 \%$ \\
\hline & & Sinduadi & 115.62 & -37.27 & $-9.67 \%$ \\
\hline & Ngaglik & Sariharjo & 119.61 & -39.84 & $-9.99 \%$ \\
\hline & & Total & $1,262.52$ & -523.53 & $-12.44 \%$ \\
\hline \multirow[t]{38}{*}{ Yogyakarta } & Danurejan & Bausasran & 10.55 & -6.91 & $-19.63 \%$ \\
\hline & & Suryatmajan & 7.25 & -2.77 & $-11.47 \%$ \\
\hline & & Tegalpanggung & 9.00 & -5.50 & $-18.34 \%$ \\
\hline & Gondokusuman & Terban & 16.71 & -10.69 & $-19.19 \%$ \\
\hline & & Baciro & 23.54 & -13.76 & $-17.54 \%$ \\
\hline & & Demangan & 15.50 & -6.06 & $-11.73 \%$ \\
\hline & & Klitren & 17.27 & -10.18 & $-17.68 \%$ \\
\hline & & Kotabaru & 15.88 & -4.61 & $-8.71 \%$ \\
\hline & Gondomanan & Prawirodirjan & 7.22 & -2.58 & $-10.72 \%$ \\
\hline & & Ngupasan & 16.56 & -5.64 & $-10.21 \%$ \\
\hline & & Prawirodirjan & 7.22 & -2.58 & $-10.72 \%$ \\
\hline & Gedongtengen & Pringgokusuman & 12.94 & -9.13 & $-21.17 \%$ \\
\hline & & Sosromenduran & 11.94 & -6.35 & $-15.96 \%$ \\
\hline & Jetis & Bumijo & 13.71 & -7.27 & $-15.91 \%$ \\
\hline & & Cokrodiningratan & 11.25 & -5.42 & $-14.45 \%$ \\
\hline & & Gowongan & 11.21 & -4.95 & $-13.26 \%$ \\
\hline & Kotagede & Prenggan & 13.25 & -5.12 & $-11.60 \%$ \\
\hline & & Rejowinangun & 16.41 & -4.21 & $-7.70 \%$ \\
\hline & & Purbayan & 6.33 & -3.16 & $-14.98 \%$ \\
\hline & Kraton & Kadipaten & 6.72 & -1.60 & $-7.16 \%$ \\
\hline & & Panembahan & 10.57 & -1.03 & $-2.91 \%$ \\
\hline & & Patehan & 6.48 & -0.78 & $-3.63 \%$ \\
\hline & Mantrijeron & Gedongkiwo & 11.27 & -5.12 & $-13.63 \%$ \\
\hline & & Mantrijeron & 12.63 & -4.23 & $-10.04 \%$ \\
\hline & & Suryodiningratan & 10.31 & -4.98 & $-14.50 \%$ \\
\hline & Mergangsan & Brontokusuman & 11.57 & -1.10 & $-2.85 \%$ \\
\hline & & Keparakan & 6.78 & -3.42 & $-15.11 \%$ \\
\hline & & Wirogunan & 12.15 & -4.35 & $-10.73 \%$ \\
\hline & Ngampilan & Ngampilan & 10.33 & -7.50 & $-21.78 \%$ \\
\hline & & Notoprajan & 6.49 & -2.44 & $-11.28 \%$ \\
\hline & Pakualaman & Gunungketur & 6.92 & -3.50 & $-15.18 \%$ \\
\hline & & Purwokinanti & 8.79 & -5.95 & $-20.29 \%$ \\
\hline & Tegalrejo & Karangwaru & 10.52 & -6.56 & $-18.71 \%$ \\
\hline & & Bener & 6.88 & -3.38 & $-14.74 \%$ \\
\hline & & Kricak & 9.11 & -6.02 & $-19.82 \%$ \\
\hline & & Tegalrejo & 9.22 & -1.65 & $-5.38 \%$ \\
\hline & Umbulharjo & Giwangan & 12.05 & -1.25 & $-3.12 \%$ \\
\hline & & Mujamuju & 19.81 & -8.59 & $-13.01 \%$ \\
\hline
\end{tabular}




\begin{tabular}{|c|c|c|c|c|c|}
\hline \multirow{2}{*}{ Regency/City } & \multirow{2}{*}{ District } & \multirow{2}{*}{$\begin{array}{c}\text { Village/Urban } \\
\text { Community }\end{array}$} & \multirow{2}{*}{$\begin{array}{c}\text { Ideal need } \\
\text { for GOS } \\
\text { (Ha) }\end{array}$} & \multicolumn{2}{|c|}{ Surplus/Deficit RTH } \\
\hline & & & & Ha & $\%$ \\
\hline & & Pandeyan & 16.74 & -1.57 & $-2.81 \%$ \\
\hline & & Semaki & 12.95 & -4.99 & $-11.57 \%$ \\
\hline & & Tahunan & 10.68 & -3.42 & $-9.59 \%$ \\
\hline & & Warungboto & 11.11 & -5.66 & $-15.28 \%$ \\
\hline & & Sorosutan & 16.99 & -3.98 & $-7.02 \%$ \\
\hline & Wirobrajan & Pakuncen & 9.19 & -2.32 & $-7.57 \%$ \\
\hline & & Patangpuluhan & 7.51 & -3.08 & $-12.30 \%$ \\
\hline & & Wirobrajan & 9.52 & -5.30 & $-16.68 \%$ \\
\hline & & Total & 527.03 & -220.65 & $-12.56 \%$ \\
\hline \multirow[t]{12}{*}{ Bantul } & Banguntapan & Banguntapan & 98.53 & -53.97 & $-16.43 \%$ \\
\hline & & Baturetno & 99.32 & -45.46 & $-13.73 \%$ \\
\hline & & Jagalan & 3.36 & -1.94 & $-17.31 \%$ \\
\hline & & Singosaren & 19.07 & 23.95 & $37.68 \%$ \\
\hline & & Tamanan & 93.68 & -71.35 & $-22.85 \%$ \\
\hline & & Wirokerten & 84.67 & -65.61 & $-23.25 \%$ \\
\hline & Kasihan & Ngestiharjo & 74.72 & -26.41 & $-10.60 \%$ \\
\hline & & Tamantirto & 103.28 & -52.83 & $-15.35 \%$ \\
\hline & & Tirtonirmolo & 77.62 & -27.96 & $-10.80 \%$ \\
\hline & Sewon & Panggungharjo & 116.95 & -70.16 & $-18.00 \%$ \\
\hline & & Bangunharjo & 173.59 & -128.98 & $-22.29 \%$ \\
\hline & & Total & 944.79 & -520.71 & $-16.53 \%$ \\
\hline \multicolumn{3}{|c|}{ Yogyakarta Urban Area (YUA) } & $2,734.34$ & $-1,264.89$ & $-13.88 \%$ \\
\hline
\end{tabular}

\section{CONCLUSIONS}

The conclusions of the study are as follow:

1. The extent of green open space in Yogyakarta urban area reached $1,469.45 \mathrm{Ha}$ or $16.2 \%$ of the total area.

2. The need for GOS was therefore lacking $23.8 \%$ of the total area.

3. The GOS condition was generally average in both housing clusters and non-housing or settlement. Good condition was located in low-density settlement, while poor GOS condition was found in high-density settlement.

\section{REFERENCES}

Baiquni, (2002), Pembangunan yang Tidak Berkelanjutan: Refleksi Kritis Pembangunan Indonesia (Unsustainable Development: Critical Review of Indonesian Development), Yogyakarta : Trans Media.

Coburn, A.W., (1994), Mitigasi Bencana (Disaster Mitigation). UNDP-Cambridge Architectural Research Limited, United Kingdom. 
Dinas Kimpraswil DIY (2006), Penyusunan RDTR Kawasan Purdowo Kabupaten Sleman (Detail Spatial Planning of Purdowo - Sleman). Laporan Akhir - Final Report. Dinas Kimpraswil DIY.

Harjito, D.A., (2007), Stock price and exchange rate causality: the case of four ASEAN countries, International Journal of Southwestern Economic Review USA, Vol. 34 (1).

Li F., Wang R., Liu X., Zhang X., (2005) Urban forest in China: Development patterns, influencing factors and research. International Journal of Sustainable Development and World Ecology; Academic Research Library. Jun 2005; 12, 2; pg. 197

Kementerian Negara Perumahan Rakyat, (2008), Peraturan Menteri Negara Perumahan Rakyat Republik Indonesia Nomor: 22/Permen/M/2008 Tentang Standar Pelayanan Minimal Bidang Perumahan Rakyat Daerah Provinsi dan Daerah Kabupaten/Kota (Regulation of State Ministry of Settlement about minimun services for Province and District level)

Reed, S. B., (1995), Pengantar tentang Bahaya (Introduction to Disaster). UNDP-Wisconsin University.

Undang-Undang Republik Indonesia Nomor 26 Tahun 2007 Tentang Penataan Ruang

Widodo B., (2005) Vulnerability Water Resources Mapping, International SURED Seminar, Integrated Water Management in Urban Environment, DAAD-Uni Karlsruhe-UKI, 2930/08/05, Jakarta

Widodo B., Lupiyanto R., Donan W., (2009a), Urban Kampong Improvement on Global Warming Mitigation Basis (A Case Study of Code River Bank Settlement Area, Yogyakarta), Proceedings of The First International Seminar on Science and Technology, Islamic University of Indonesia (UII) - Universiti Kebangsaan Malaysia - Universiti Malaysia Trengganu, January 24-25, 2009

Widodo B., Lupiyanto R., Donan W., (2009b), Model dan Strategi Penguatan Kapasitas Sumberdaya Air dalam Mendukung Pertumbuhan Sosial Ekonomi Kabupaten Bantul Provinsi DIY (Strategy for improving water resources capacity for supporting soio economic development in Bantul, DIY), Laporan Hibah Kompetitif Penelitian Kerjasama Internasional dalam rangka Publikasi Internasional DIKTI, Yogyakarta: Universitas Islam Indonesia

Widodo B., R. Lupiyanto, Donan W., J. Hamidin, (2011), Urban Heat Islands Mitigation by Green Open Space (GOS) Canopy Improvement: A Case of Yogyakarta Urban Area (YUA), Indonesia, International Journal of Technology, Vol 2, No 3 (2011), http://www.ijtech.eng.ui.ac.id/index.php/journal/article/view/69 